\title{
STRESS AND FATIGUE IN OPERATORS UNDER RADIOFREQUENCY ELECTROMAGNETIC RADIATION AND SHIFT WORK
}

\author{
K. Vangelova and D. Velkova \\ National Center of Public Health and Analyses
}

Summary. The aim was to study the effect of radiofrequency electromagnetic radiation $(E M R)$ on stress indices, health complaints and fatigue of operators working fast-rotating extended shifts. Working conditions, job content, job control, social support, health complaints and fatigue were followed in 220 operators, 110 exposed to EMR and 110 control operators, matched by age and sex. The EMR was measured and time-weighted average (TWA) was calculated. The excretion rates of stress hormones cortisol, adrenaline and noradrenaline were followed during the extended shifts in 36 operators, working at different levels of exposure and 24-hour exposure was calculated.The exposed group pointed more problems with the working conditions, including EMR, noise, currents and risk of accidents, more health complaints and higher level of fatigue. The most common health complaints were mental and physical exhaustion after work, pains in the chest, musculoskeletal complaints, headache, and apathy. High level EMR exposure $\left(T_{\text {TWA }}=3.10 \mu \mathrm{W} / \mathrm{cm}^{2}\right.$, TWA $_{\max }=137.00 \mu \mathrm{W} / \mathrm{cm}^{2}$ ) significantly increased the 24-hour excretion of cortisol and noradrenaline, whereas the increase of adrenaline excretion did not reach significance, as well as hormone excretion rates under low level exposure $\left(\mathrm{TWA}_{\text {mean }}=1.89 \mu \mathrm{W} / \mathrm{cm}^{2}, \mathrm{TWA}_{\max }=5.24 \mu \mathrm{W} / \mathrm{cm}^{2}\right.$ ). In conclusion, higher number of health complaints, higher stress hormone excretion rates and fatigue were found in operators under EMR. fatigue.

Key words: EMR exposure, radiofrequency range, shiftwork, health complaints, stress,

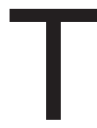
he technical staff in broadcasting (BC) and TV stations is working under monotony, extended shifts and radiofrequency electromagnetic radiation (EMR). The monotonous tasks, the shift work, the long working hours are known health risk factors. Shift work is related to a higher risk of sleep disturbances, fatigue and ill health, especially when night work is required $[1,5,10]$. 
The health risks of radiofrequency EMR exposures have been studied, and besides still controversies exist, variety of health conditions have been described [6, $15,16]$. Our earlier data showed higher rates of cardiovascular risk factors and exposure-relationship with the stress hormones under radiofrequency EMR $[8,19,20]$. In some studies no effect on stress hormones of radiofrequency EMR was found [2, 7].

Non-specific health symptoms are described under different radiofrequency exposures $[6,12,14]$, the main are headaches, fatigue, sensations of warmth, etc., but clear evidence of a causal relationship between radiofrequency EMR and such symptoms, however, is lacking. In occupational settings, the combination of variety risk factors can exaggerate to higher effect and more health symptoms.

The aim was to study the effect of radiofrequency electromagnetic radiation (EMR) on stress indices, health complaints and fatigue of operators working fast-rotating extended shifts.

\section{MATERIAL AND METHODS}

The study covers 220 operators, 110 exposed radiofrequency EMR and 110 control operators, matched by age and sex. The group of exposed operators consists of two groups exposed to different levels of radiofrequency EMR: I group - 49 persons ( 35 males and 14 females) from a BC station $(6-25 \mathrm{MHz})$ of age $47.9 \pm 6.7$ years and length of service $25.7 \pm 5.5$ years; II group -61 persons (53 males and 8 females) from four TV stations ( $66 \mathrm{MHz}-900 \mathrm{MHz}$ ) of age $47.9 \pm 7.0$ years and length of service $25.9 \pm 7.5$ years. All subjects have signed informed consent.

The EMR exposure was assessed by measuring the intensities and flux densities of the electromagnetic field at the typical work places of the staff according to the duty records for the near zone with NFM-1 (Germany) and for the far zone with RAHAM (USA), as earlier described [20]. The intensities and the flux densities of the EMF were within ICNIRP limits [9], except for the antenna field of the BC station, where the eventual stay of the operators was very limited. In order to be able to compare the electromagnetic exposure in the studied stations the intensities in the short wave range were transformed into flux densities for flat electromagnetic field. The TWA was calculated. The calculations included the mean and maximum TWA. The TWAmean for BC station operators showed exposure in the range $3.10-3.96$ $\mu \mathrm{W} / \mathrm{cm}^{2}$ and TWAmax in the range from 66.90 to $137 \mu \mathrm{W} / \mathrm{cm}^{2}$. The TWAmean of TV station operators showed exposure from 1.19 to $1.89 \mu \mathrm{W} / \mathrm{cm}^{2}$ and TWAmax from 1.28 to $5.24 \mu \mathrm{W} / \mathrm{cm}^{2}$ [20]. EMR at both typical constant and temporary work places of the radio-relay station operators (control group) were not detectable. The noise and microclimate were followed and were within hygienic norms with no differences between the groups.

Psychosocial factors were assessed using the My Job questionnaire [11] containing five subscales. The working conditions scale contains items on working pose, 
lightening, noise, vibrations, temperature, humidity, flow, dust, odours, etc. The job content scale sums 18 items (monotony, tasks, requiring intense concentration, time pressure, work organization, etc.). The job control scale (10 items) includes questions on novelties at work, ability to influence pace, methods of work, professional realization, etc.). The work-related social support sums 10 items: 5 concerning support from co-workers and 5 from supervisors. The health complaints scale is the sum of 10 items.

The chronic fatigue was followed using the Checklist of Individual Strength [3]. The questionnaire consists of 20 statements, testing different aspects of fatigue: subjective feeling of fatigue, reduced motivation, reduced activation, and reduced concentration. The answers of the four scales are summed to estimate chronic fatigue.

The excretion rates of stress hormones cortisol, adrenaline and noradrenaline were followed during 24-hour period, including one extended shift, in 36 operators, working at different levels of exposure. The operators were asked to void at 09:00 and urine samples were collected at 4-hour interval and stored at $-20^{\circ} \mathrm{C}$. Before refrigeration the subsamples for catecholamines were acidified to $\mathrm{pH} 3$ with $\mathrm{HCl}$. Urine free cortisol was assessed with an RIA kit (Orion Diagnostica, Espoo, Finland, the interassay CV was 5.2, 5.2, 6.1 for low, medium and high value urine samples. Adrenaline and noradrenaline were measured with spectrofluorimetry [18] and 24hour excretion was calculated.

The data were analysed with one-way ANOVA, correlation and stepwise multiple regression analysis using SPSS software.

\section{RESULTS}

Our data show that working conditions were described as nearly good (Figure 1), except for the negative ratings for EMR, noise, currents and risk of accidents, contributing to significantly higher number of negative ratings with the EMR-exposed group (Table 1). During the study the operators were not aware of the EMR. Work tasks were considered as monotonous and required concentration in both groups. The job control and social support were good with all studied. Most of the inquired felt insecure for their work places; they felt a treat of reorganizations and being fired. Fear of job loss can be viewed as general stressor for both EMR-exposed and control group. The exposed group showed higher rates of health complaints. The main health complaints were physical exhaustion after work, pains in the chest, pain in the muscles, bones and back, fatigue, apathy, sleep problems, more often in EMFexposed group.

Concerning fatigue only the scale of subjective fatigue reached significance with more pronounced feeling of fatigue of EMR-exposed group, and the total sum was within a trend. The correlations between different scales of the questionnaire 
"My job" and chronic fatigue are common for both groups, but with higher significance in the control group (Table 3).

Table 1. Positive ratings on the My Job questionnaire of EMF-exposed and control operators

\begin{tabular}{|c|c|c|}
\hline \multirow{2}{*}{ Questionnaire scales and indices } & \multicolumn{2}{|c|}{$\%$ of positive answers } \\
\hline & EMR-exposed & Control group \\
\hline \multicolumn{3}{|l|}{ Working conditions } \\
\hline Noise & 72.9 & 50.0 \\
\hline Currents & 59.3 & 33.8 \\
\hline EMR & 81.9 & 65.2 \\
\hline Risk of accident & 65.1 & 45.9 \\
\hline \multicolumn{3}{|l|}{ Job content } \\
\hline Monotonous tasks & 52.3 & 46.2 \\
\hline Tasks, requiring intensive concentration & 83.5 & 68.8 \\
\hline Work under time pressure & 58.1 & 46.9 \\
\hline Isolated work places & 19.6 & 40.5 \\
\hline Problems with the equipment & 52.9 & 44.4 \\
\hline \multicolumn{3}{|l|}{ Job control } \\
\hline Insufficient opportunity for professional advancement & 43.1 & 50.0 \\
\hline Insecurity of the work places & 74.4 & 72.9 \\
\hline Job satisfaction & 92.2 & 90.1 \\
\hline \multicolumn{3}{|l|}{ Health complaints } \\
\hline Physical exhaustion after work & 55.3 & 33.8 \\
\hline Pains in the chest & 52.3 & 42.2 \\
\hline Pain in the muscles and bones & 70.6 & 40.6 \\
\hline Pain in the back & 56.9 & 53.8 \\
\hline Fatigue & 46.2 & 40.0 \\
\hline Apathy & 55.8 & 35.4 \\
\hline Sleep problems & 45.2 & 36.0 \\
\hline
\end{tabular}


Table 2. Mean values ( $x, S D)$ of the scales of questionnaire of chronic fatigue of EMF-exposed and control group

\begin{tabular}{|c|c|c|c|c|c|c|c|c|c|c|}
\hline Grou & $\begin{array}{c}\text { Subjective } \\
\text { fatigue }\end{array}$ & $\mathrm{p}$ & $\begin{array}{c}\text { Reduced } \\
\text { motivation }\end{array}$ & $\mathrm{p}$ & $\begin{array}{c}\text { Reduced } \\
\text { activity }\end{array}$ & $\mathrm{p}$ & $\begin{array}{c}\text { Reduced } \\
\text { concentration }\end{array}$ & $\mathrm{p}$ & $\begin{array}{l}\text { Total } \\
\text { fatigue }\end{array}$ & p \\
\hline Ration & $5.5(11.3)$ & \multirow[t]{2}{*}{0.03} & $0(4.0)$ & \multirow[t]{2}{*}{0.29} & & \multirow[t]{2}{*}{0.71} & $10.02(5.0)$ & \multirow[t]{2}{*}{0.94} & $52.4(17.9)$ & \multirow[t]{2}{*}{0.09} \\
\hline Control group & 21.4 (11.4) & & $8.5(4.1)$ & & $7.3(4.1)$ & & $9.9(5.4)$ & & $47.3(18.9)$ & \\
\hline
\end{tabular}

Table 3. Significant correlations between the psychosocial factors at workplace and chronic fatigue scales for EMR-exposed and control group

\begin{tabular}{|c|c|c|c|c|c|c|}
\hline Scales & Groups & Subjective fatigue & Motivation & Activity & Concentration & Total \\
\hline \multirow{2}{*}{ Working conditions } & exposed & & & & & \\
\hline & control & & $0.32^{*}$ & & $0.38^{\star *}$ & $0.41^{\star \star}$ \\
\hline \multirow{2}{*}{ Job content } & exposed & $0.28^{\star}$ & & & & \\
\hline & control & $0.30^{*}$ & & & & $0.31^{*}$ \\
\hline \multirow{2}{*}{ Job control } & exposed & $0.24^{*}$ & $0.24^{*}$ & & & $0.24^{*}$ \\
\hline & control & $0.36^{*}$ & $0.59^{\star \star \star}$ & $0.39 *$ & $0.58^{\star \star \star}$ & $0.58^{\star \star \star}$ \\
\hline \multirow{2}{*}{ Social support } & exposed & $0.28^{* \star}$ & & & & $0.26^{*}$ \\
\hline & control & $0.36^{*}$ & $0.48^{* \star}$ & & $0.51^{\star \star \star}$ & $0.49^{\star \star *}$ \\
\hline \multirow{2}{*}{ Health complaints } & exposed & $0.66^{\star \star *}$ & $0.43^{\star \star *}$ & & $0.46^{* * *}$ & $0.69^{* * \star}$ \\
\hline & control & $0.43^{* *}$ & $0.98^{\star \star \star}$ & $0.74^{\star \star \star}$ & $0.98^{\star * \star}$ & $0.90^{\star * \star}$ \\
\hline
\end{tabular}

${ }^{*} p<0.05 ;{ }^{* *} p<0.01 ;{ }^{* * *} p<0.001$

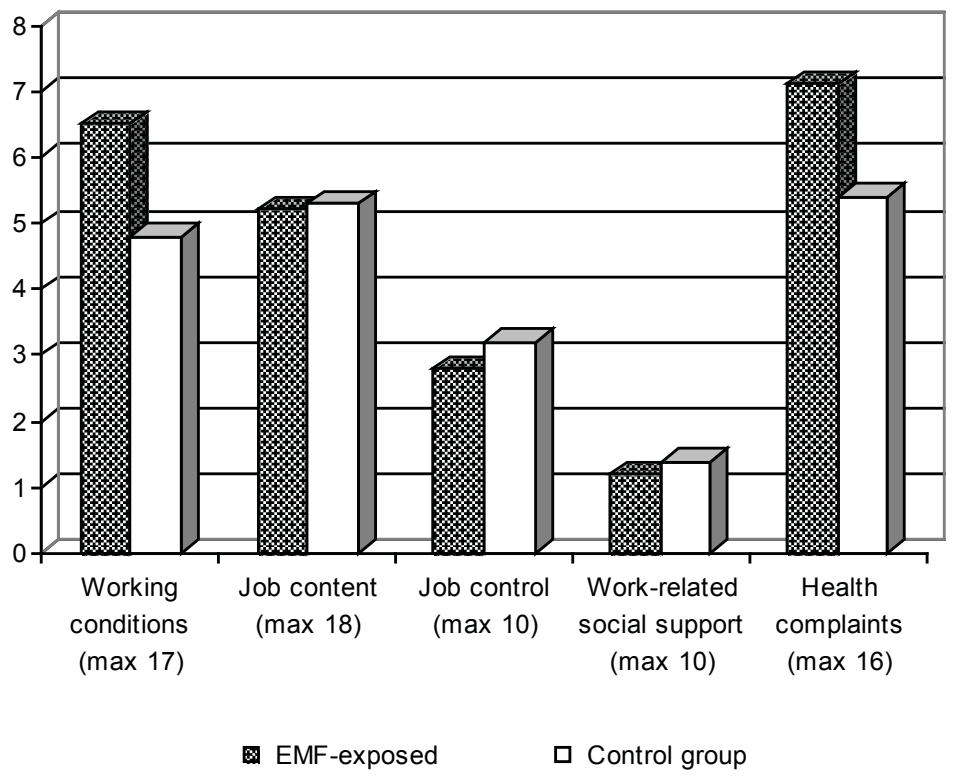

Fig. 1. Psychosocial factors and health complaints in EMF-exposed and control operators 
High level EMR exposure $\left(\mathrm{TWA}_{\text {mean }}=3.10 \mu \mathrm{W} / \mathrm{cm} 2, \mathrm{TWA}_{\max }=137.00 \mu \mathrm{W} / \mathrm{cm}^{2}\right)$ significantly increased the 24-hour excretion of cortisol and noradrenaline (Table 4), where as the increase of adrenaline excretion did not reach significance, as well as hormone excretion rates under low level exposure $\left(\mathrm{TWA}_{\text {mean }}=1.89 \mu \mathrm{W} / \mathrm{cm}^{2}\right.$, $\left.\mathrm{TWA}_{\max }=5.24 \mu \mathrm{W} / \mathrm{cm}^{2}\right)$. The 24-hour excretion significantly correlated with the TWA $_{\text {mean }}(r=0.343, p=0.047$ and $r=0.386, p=0.024$ for cortisol and noradrenaline $)$ and TWA max $_{\text {m }}(r=0.339, p=0.05$ and $r=0.356, p=0.038$ for cortisol and noradrenaline).

Table 4. The 24-hour excretion of cortisol, adrenaline and noradrenaline of operators exposed to high- and low-level EMR radiation and control group

\begin{tabular}{|l|c|c|c|}
\hline Indices/Groups & Cortisol $(\mathbf{n m o l} / \mathbf{2 4} \mathrm{h})$ & Adrenaline $(\mathrm{nmol} / \mathbf{2 4} \mathrm{h})$ & Noradrenaline $(\mathrm{nmol} / \mathbf{2 4} \mathrm{h})$ \\
\hline High-level exposure & $160.94 \pm 86.72^{\star \star}$ & $39.83 \pm 23.31$ & $174.14 \pm 71.30^{\star}$ \\
\hline Low-level exposure & $126.95 \pm 71.72$ & $36.14 \pm 14.12$ & $136.30 \pm 31.74$ \\
\hline Control group & $88.62 \pm 45.25$ & $25.78 \pm 10.94$ & $116.91 \pm 40.93$ \\
\hline
\end{tabular}

${ }^{*} \mathrm{~F}=4.773, \mathrm{p}=0.041$; ** $\mathrm{F}=5.561, \mathrm{p}=0.023$ for high-level exposure/control operators

\section{DISCUSSION}

The EMR-exposed group pointed more problems with working conditions, including EMR, noise, currents and risk of accidents, but as a whole the working conditions were described as good by both groups. More health complaints and higher level of fatigue was also rated by the EMR-exposed group. The most common health complaints were mental and physical exhaustion after work, pains in the chest, musculoskeletal complaints, headache, apathy and sleep disorders. Some of the health complaints are comparable with other studied groups [17]. Our data showed weaker correlations between the psychosocial factors and fatigue in EMR-exposed operators in comparison to the control group. Any interpretation would be speculative, but the shift work schedules and rest periods arrangements during work showed no considerable differences between the two groups.

Our data show higher 24-hour cortisol, adrenaline and noradrenaline excretion with higher EMR exposure operators, with a significantly higher cortisol and noradrenaline 24-hour excretion in high-level exposure operators in comparison to control group. These data confirm our previous data [20], but further research is needed with better control of confounding factors. As stress hormones were studied only in small groups, our data do not allow us to search relations with fatigue and 
health complaints, but higher stress levels are known to contribute both to ill health and fatigue $[4,13]$.

In conclusion, higher number of health complaints, higher stress hormone excretion rates and fatigue were found in operators under EMR. The combination of exposure to radiofrequency EMR, shiftwork and monotony raises concerns for health and safety of the employees, preventive actions should be undertaken as reduction of radiofrequency EMR exposure to as low as possible, implementation of ergonomic shiftwork schedules and adequate rest periods arrangements during work.

\section{REFERENCES}

1. Akerste dt, T. Shift work and disturbed sleep/ wakefulness. - Occup. Med., 53, 2003, 89-94.

2. Bortkiewicz, A. et al. Subjective symptoms reported by people living in the vicinity of cellular phone base stations: review. - Med. Pr., 55, 2004, 4, 345-351.

3. Bultmann, U. Fatigue and Psychological distress in working population. The role of work and lifestyle. - PhD Thesis. Datawyse/Universitat Maastricht, 2002, pp.141.

4. Chrousos, G. P. The role of stress and the hypothalamic-pituitary-adrenal axis in the pathogenesis of metabolic syndrome: neuroendocrine and target tissue-related causes. - Intern. J. Obesity, 24, 2000, Suppl. 2, S50-55.

5. Costa, G. Shift work and occupational medicine: an overview. - Occup. Med., 53, 2003, 83-88.

6. J a u c h e m, J. Effects of low-level radiofrequency $(3 \mathrm{kHz}$ to $300 \mathrm{GHz})$ energy on human cardiovascular, reproductive, immune and other systems: A review of the recent literature. - Int. J. Hygiene and Environmental Health, 211, 2008, № 1-2, 1-29.

7. Hutter, H. Subjective symptoms, sleeping problems and mobile phone base stations. - Occup. Environ. Med., 63, 2006, 307-313.

8. Is rae I, M., Vangelova K., Ivanova M. Cardiovascular risk in physiotherapists under electromagnetic radiation. - Environmentalist 27, 2007, № 4, 539-543.

9. ICNIRP. Guidelines for limiting exposure to time-varying electric, magnetic and electromagnetic fields (up to $300 \mathrm{GHz}$ ). - Health Physics, 74, 1998, № 4, 494-522.

10. Knuts s on, A. Health disorders of shift workers. - Occup. Med., 53, 2003,103-108.

11. Kompier, M. et L. Levi. Stress at work: causes, effects and prevention. A guide for small and medium sized enterprises. - European Foundation for Improvement of Living and Working Conditions. 1994, Geneva.

12. Mann, K. et al. Effects of pulsed high frequency electromagnetic fields on the neuroendocrine system. - Neuroendocrinology, 67, 1998, 139-144.

13. McEwen, B. S. Protective and damaging effects of stress mediators. - N. Engl. J. Med., 338, 1998, № 3, 171-179.

14. Randon, K. et al. No effects of pulsed radiofrequency electromagnetic fields on melatonin, cortisol and selected markers of the immune system in men. - Bioelectromag, 22, 2001, 280-287.

15. Rö ö sli , M., R. Rapp et C. Braun-Fahrlander. Radio and microwave frequency radiation and health - An Analysis of the literature. - Gesundheitswesen, 65, 2003, № 6, 378-392. 
16. Rö ös II, M. et al. Systematic review on the health effects of exposure to radiofrequency electromagnetic fields from mobile phone base stations. - Bull. World Health Organ., 88, 2010, № 12, 887-896.

17. Tzenova, B. Stress and psychosocial risk factors. - Bulg. J. Publ. Health, 2, 2010, № 2, 23-29 (in $\mathrm{Bg}, \mathrm{En}$ ).

18. Vang e lova, K. Method for assessment of catecholamines adrenaline and noradrenaline in urine. - In: Methods for Investigations in Hygiene. National Center of Hygiene, Medical Ecology and Nutrition, Sofia, 2000, № 2, 2-4 (in Bulgarian).

19. Vangelova, K., Deyanov C. et Israel M. Cardiovascular risk under radiofrequency electromagnetic radiation. - Int. J. Hyg. Environ. Health, 209, 2006, 133-138.

20. Vangelova, K. et Israel M. Variations of melatonin and stress hormones under extended shifts and radiofrequency electromagnetic radiation. - Rev. Environ. Health, 20, 2005, № 2, 151-161.

\section{Corresponding author:}

Katia Vangelova

National Center of Public Health and Analyses

15 Akad. Ivan Geshov Blvd.

$\mathrm{Bg}-1431$ Sofia

(D) +35928056224

e-mail: k.vangelova@ncpha.government.bg 